\title{
IMPACT OF HANDGRIP EXERCISE INTENSITY ON
}

\section{BRACHIAL ARTERY FLOW-MEDIATED DILATION}

\author{
Atkinson, Ceri L. ${ }^{1}$ \\ Carter, Howard H. ${ }^{1}$ \\ Dawson, Ellen A. ${ }^{2}$ \\ Naylor, Louise H. ${ }^{1}$ \\ Thijssen, Dick H.J. ${ }^{2,3}$ \\ Green, Daniel J. ${ }^{1,2}$
}

${ }^{1}$ School of Sports Science, Exercise and Health

University of Western Australia, Crawley, Western Australia

${ }^{2}$ Research Institute for Sport and Exercise Science

Liverpool John Moores University, Liverpool, United Kingdom

${ }^{3}$ Department of Physiology,

Radboud University Nijmegen Medical Centre, Nijmegen, Netherlands

\section{Running Head:}

Exercise intensity and endothelial function

\section{Author for correspondence:}

Ceri Atkinson

School of Sport Science, Exercise and Health,

The University of Western Australia, Crawley, Western Australia, 6009

Phone: +61 (8) 6488 2378, Fax: +61 (8) 64881039

Email: ceri.atkinson@research.uwa.edu.au 


\section{Abstract}

2 Purpose. Previous studies that have examined the impact of exercise intensity on conduit

3 artery endothelial function have involved large muscle group exercise which induces local

4 and systemic effects. The aim of this study was to examine flow mediated dilation (FMD)

5 before and after incremental intensities of handgrip exercise (HE), to assess the role of local

6 factors such as blood flow and shear rate on post-exercise brachial artery function. Methods.

7 Eleven healthy men attended the laboratory on 3 occasions. Subjects undertook 30 minutes of

8 handgrip exercise at 3 intensities $(5,10$ or $15 \%$ MVC). Brachial artery FMD, shear and blood

9 flow patterns were examined before, immediately after, and 60 minutes post exercise.

10 Results. Handgrip exercise increased mean and antegrade shear rate (SR) and blood flow

11 (BF), and reduced retrograde SR and BF (all $P<0.01$ ). Exercise intensity was associated with

12 a dose-dependent increase in both mean and antegrade BF and SR (interaction, $P<0.01$ ).

13 Post-hoc tests revealed that, whilst handgrip exercise did not immediately induce post-

14 exercise changes, FMD was significantly higher 60 minutes post-exercise following the

15 highest exercise intensity $(5.9 \pm 2.8$ to $10.4 \pm 5.8 \%, P=0.01)$. Conclusions. HE leads to

16 intensity-and time-dependent changes in conduit artery function, possibly mediated by local

17 increases in shear, with improvement in function evident at 1 hour post-exercise when

18 performed at a higher intensity. 


\section{Introduction}

It is well established that exercise training reduces cardiovascular risk (Green et al. 2008), in part due to the direct effects of exercise on the function and health of the vessel wall (Green 2009; Joyner and Green 2009; Laughlin and McAllister 1992). Episodic exercise-induced increases in blood flow, shear stress and perhaps transmural pressure are now recognized as important physiological stimuli for training-based improvements in vascular health (Laughlin et al. 2008; Tinken et al. 2009). Despite the chronic effect of exercise training on the vascular endothelium being well studied, the acute impacts of distinct forms and intensities of exercise are less well described.

Vascular function is commonly assessed using the flow-mediated dilation (FMD) technique, a partially nitric oxide- and endothelium-dependent (Green et al. 2014) conduit artery response to a brief ischemic stimulus (Corretti et al. 2002; Thijssen et al. 2011) which predicts cardiovascular events (Gocke et al. 2003; Takase et al. 1998). Studies of the FMD response to acute exercise have reported increases (Johnson et al. 2012; Tinken et al. 2009; Tyldum et al. 2009; Zhu et al. 2010), decreases (Dawson et al. 2008; Goel et al. 2007; Jones et al. 2010; Llewellyn et al. 2012; Rognmo et al. 2008), or no change (Dawson et al. 2008; Harvey et al. 2005; Jones et al. 2010). These inconsistencies have been linked to methodological differences between studies, the timing of post-exercise FMD measures and the possibility of intensity and modality-specific exercise effects (Dawson et al. 2013). A recent study conducted by Birk and colleagues (Birk et al. 2013) addressed some of these issues by assessing FMD at multiple time-points following 30 minutes of lower limb cycle exercise of differing intensities. A 'biphasic' response pattern, characterized by an initial post-exercise FMD reduction, followed by a normalization 60 minutes post-exercise, was observed. Furthermore, the reduced FMD following cycle exercise was intensity dependent, 

61

with larger reductions observed following higher exercise intensity (Birk et al. 2013). These changes in FMD may be related to the pattern of blood flow and shear stress through the brachial artery during cycling, but endocrine and reflex effects cannot be excluded, given the systemic nature of exercise stimulus.

The primary aim of the present study was to describe the time-dependent effects of handgrip exercise, performed at different exercise intensities, on FMD immediately post-exercise and 60-minutes following exercise cessation. Our rationale for adopting handgrip exercise, which utilizes a small muscle mass, was that it may provide insight into whether localized mechanisms (particularly those related to changes in blood flow and shear stress) contribute to the acute effects of exercise on arterial function. We hypothesized that an intensitydependent decrease in FMD would occur immediately after exercise, with higher intensities of handgrip exercise leading to a larger post-exercise reduction in FMD, followed by a return of function to baseline levels after 60 -minutes of recovery.

Keywords: exercise intensity; endothelial function; shear stress; cardiovascular risk 
63 Abbreviations:

64 ANOVA: analysis of variance

65 BF: blood flow

66 DBP: diastolic blood pressure

67 FMD: flow mediated dilation

68 HE: handgrip exercise

69 LMM: linear mixed model

70 LSD: least significant difference

71 MAP: mean arterial pressure

72 MVC: maximal voluntary contractile

73 ROS: reactive oxygen species

74 SBP: systolic blood pressure

75 SR: shear rate

76 


\section{Methods}

\section{Participants}

Eleven healthy male subjects (age: $27 \pm 3$ years, height: $1.78 \pm 0.08 \mathrm{~m}$ weight: $75.2 \pm 11.5 \mathrm{~kg}$ ) volunteered for the study. Women were excluded due to the cyclical effects of estrogen on vascular function (Williams et al. 2001). Based on detailed medical questionnaires, we excluded subjects with pre-existing medical conditions (including cardiovascular diseases) and cardiovascular risk factors such as hypertension, hypercholesterolemia and type 1 or 2 diabetes, as well as those taking any medications. The study was approved by the University of Western Australia's institutional ethics committee and adhered to the Declaration of Helsinki. Informed consent was obtained from all participants before undertaking experimental procedures.

\section{Experimental Design}

Participants attended all testing sessions in a fasted state ( $>6 \mathrm{hrs}$ ), having avoided strenuous exercise, alcohol and caffeine for 18 hours prior to arrival (Thijssen et al. 2011). Participants undertook 3 separate experimental sessions over two weeks, with a minimum of 48 hours between sessions. Upon arrival at the laboratory, participants were required to complete an assessment of maximal voluntary contractile (MVC) handgrip strength. This involved three maximal contractions, separated by 5 minute rest intervals, using a grip strength dynamometer. An average of the three contractions was used to calculate MVC which was used in subsequent sessions to set exercise intensities. After a 20 minute supine rest period on a bed, subjects performed 30 minutes of unilateral handgrip exercise (HE) at either 5,10 or $15 \%$ of maximal voluntary handgrip contraction strength (MVC) at a rate of 30 isotonic contractions a minute, based on similar protocols adopted by our research team (Tinken et al. 2009). The order of testing was randomised between subjects. The arm selected to undertake 
the 30 minute exercise stimulus (preferred vs non-preferred) was also randomised between subjects, although we maintained the same arm within subjects across all three sessions. Brachial arterial endothelial function was assessed prior to, immediately after, and 60minutes following cessation of handgrip exercise using the flow mediated dilation (FMD) technique (Thijssen et al. 2011). We also continuously recorded brachial artery diameter, blood flow and shear rate throughout each HE session. Finally, blood pressure was continuously monitored throughout the exercise duration (Finapres Medical Systems, Netherlands),

\section{Experimental Measurements}

\section{Assessment of brachial artery endothelial function}

After a 20-minute rest period, brachial artery endothelial function was assessed using the flow-mediated dilation (FMD) technique in accordance with recently published guidelines (Thijssen et al. 2011). Briefly, the arms were extended to an $\sim 80^{\circ}$ angle from the torso. A rapid inflation/deflation pneumatic cuff (D.E. Hokanson, Bellevue, WA) was placed around the forearm, distal to the olecranon process. Using a $10-\mathrm{MHz}$ multi-frequency linear array probe, attached to a high-resolution ultrasound machine (T3000; Terason, Burlington, MA), an optimal B-mode image of the brachial artery was acquired. Settings were optimized, recorded and used for subsequent repeat sessions. Following image acquisition, one minute of baseline imaging was performed, after which the forearm cuff was inflated to $220 \mathrm{mmHg}$ for 5 minutes. Thirty seconds prior to cuff deflation, recordings of diameter and velocity resumed and were continued for 3 minutes following cuff deflation, in accordance with previous studies (Woodman et al. 2001). This procedure was repeated immediately following cessation of the handgrip exercise, and again 60-minutes thereafter. 


\section{Brachial artery diameter and blood flow analysis}

Brachial artery diameter and velocity were analysed using custom-designed edge-detection and wall-tracking software, which is largely independent of investigator bias (Woodman et al. 2001). Specific details of analysis techniques are described elsewhere (Thijssen et al. 2009a). Continuous $(30 \mathrm{~Hz})$ diameter and velocity data were used to calculate blood flow (the product of lumen cross-sectional area and Doppler velocity). Shear rate was also calculated as 4 times mean blood velocity/vessel diameter. Reproducibility of diameter measurements using this semi-automated software is significantly better than manual methods, reduces observer error significantly, and possesses an intra-observer coefficient of variation of $6.7 \%$ (Woodman et al. 2001). FMD was calculated in absolute $(\mathrm{mm})$ and relative $(\%)$ terms as the increase from the resting baseline diameter, as described in detail in previously (Thijssen et al. 2011). The shear rate stimulus to FMD, SRAUC, was calculated in each individual by plotting SR collected at $30 \mathrm{~Hz}$ continuously from the time of cuff deflation to the time of peak diameter attainment, and then summing the areas of successive post-occlusion trapezoids (each with a base of $3-\mathrm{sec}$ ). This approach is consistent with literature best practice and the body of literature on this topic, largely pioneered by Tschakovsky and Pyke (Thijssen et al. 2011). We also analysed brachial artery diameter and velocity data across 30 -second epochs, at 5 minute intervals, throughout the 30 minute unilateral handgrip exercise bout. In the present study, all files were coded such that the individual undertaking the automated analysis was nonetheless blinded to each subject's identity and the exercise intensity at which the file in question was collected.

\section{Statistics}

Statistical analysis was performed using SPSS 21.0 (SPSS, Chicago, IL) software. A two-way repeated measures ANOVA was used to compare changes in FMD\%, SR, blood pressure 
152

153

154

155

156

157

158

159

160

161

162

163

164

165

166

167

168

169

170

171

172

173

174

175

across 'time' (pre, post \& 60mins post) and whether these changes occurred across differing 'intensity' bouts $(5,10 \& 15 \%$ MVC). Post-hoc analysis was performed using the least significant difference (LSD) method. All data are reported as mean $( \pm \mathrm{SD})$ unless stated otherwise and statistical significance was assumed at $\mathrm{P}<0.05$. Additional statistics were adopted in order to account for influences known to affect FMD responses, principally arterial diameter and $\mathrm{SR}_{\mathrm{AUC}}$ changes (Atkinson et al. 2013). To do so, we analysed the effects of intensity and time, as well as the interaction effect for FMD, on the change in logarithmically transformed diameter using a Linear Mixed Model (LMM). This model incorporated baseline arterial diameter and shear rate area under the curve as covariates, where these baseline arterial diameter data were specific to each FMD test. This sample size was similar to those reported in previous studies on the effects of exercise (Johnson et al. 2012; Padilla et al. 2006; Padilla et al. 2011), and we estimated that a $2 \%$ change in FMD would be detected with 10 participants assuming that the standard deviation of this change is $2 \%$ with statistical power of $80 \%$ (Birk et al. 2013).

6

\section{Results}

There were no differences in resting (pre-exercise) brachial diameter, FMD\%, FMDmm, shear rate or $\mathrm{SR}_{\mathrm{AUC}}$ across the three testing days $(P>0.05)$ (Table 1). Participants completed 30 minutes of isotonic $\mathrm{HG}$ exercise (30 contractions per minute) at each exercise intensity. Mean arterial pressure (MAP), systolic blood pressure (SBP), diastolic blood pressure (DBP) and heart rate all increased in an exercise intensity "dose-dependent" manner (interactioneffect: all $P<0.05$ ), with the largest elevations seen during 30 minutes of HG exercise at $15 \%$ MVC (Table 2). 


\section{Brachial artery blood flow and diameter during handgrip exercise}

178 Unilateral handgrip exercise resulted in an immediate increase in both mean and antegrade blood flow and shear rate, which remained elevated throughout the 30-minute exercise bout (Figure 1a). Higher exercise intensity was associated with a larger increase in both mean and antegrade blood flow and shear rate across the exercise duration (interaction-effect: $P<0.01$ ). Post-hoc t-tests using an LSD approach revealed differences at all-time points of exercise for antegrade SR at $15 \%$ MVC compared to both $10 \%$ (all $P<0.01$ ) and 5\% (all $P<0.01$ ). Retrograde shear rate during each 30 minute bout of handgrip exercise was reduced (time effect: $P=<0.01$ ), although this reduction did not significantly differ between intensities (interaction-effect: $P=0.07$, Figure $1 b)$. Exercise at a low intensity (5\% MVC) did not significantly alter brachial artery diameter following exercise $(P=0.64$, Table 3$)$, whereas $10 \%$ MVC evoked a decrease in diameter 60 minutes post-exercise $(P=0.04)$. At $15 \%$ MVC, diameter increased immediately following exercise $(P=0.01)$, but had returned to baseline values at 60 minutes $(P=0.16)$.

\section{Brachial artery flow-mediated dilation}

Two-way ANOVA revealed a significant time*intensity interaction-effect $(P=0.01)$ for FMD\% (Table 3). Post-hoc analysis revealed a significant increase in FMD 60-minutes after the $15 \%$ MVC handgrip exercise bout $(P<0.01)$, whilst no change in FMD was observed during at other time-points or exercise intensities. Similar changes were observed for absolute FMD, with a significant interaction-effect $(P<0.01)$ and a significant increase in FMD 60minutes after 15\% MVC handgrip exercise session (Table 3). Given that FMD responses are inversely proportional to baseline arterial diameter (Thijssen et al. 2008), the interaction 
207

effect observed for FMD\% may have been influenced by acute exercise impacts on baseline arterial diameter (Birk et al. 2013; Dawson et al. 2013; Padilla et al. 2007). In order to account for potential impacts of baseline diameter, we undertook the LMM for FMD with baseline diameter as a covariate. This analysis reinforced our initial findings and revealed a significant interaction effect between time (pre, post, 60-post) and exercise intensity $(5,10$ $\& 15 \% \mathrm{MVC})(P=0.03)$

The tabulated FMD\% data are summarized in Figure 2, in which change in FMD (expressed as the immediate post-exercise FMD\% minus baseline pre-exercise FMD\%) was not significantly different between exercise intensities ( $P=0.67$, Figure $2 \mathrm{~A})$. However, change in FMD, 60 minutes post-exercise vs baseline, showed a dose-response relationship with exercise intensity $(P<0.01)$, with a $0.7 \%$ decreased FMD at the lowest exercise intensity, and a $4.5 \%$ increased FMD following $\mathrm{HG}$ exercise at the highest intensity (t-test: $P=0.01$, Figure 2B).

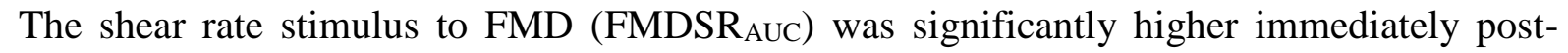
exercise under each of the three conditions (Table $3 P=<0.01$ ), however, it had returned to pre-exercise levels by the time the 60-minutes post-exercise FMD test was performed (Table 3). There were no differences in FMD $\mathrm{SR}_{\mathrm{AUC}}$ between the exercise conditions $(P=0.11)$. Statistically accounting for changes in $\mathrm{SR}_{\mathrm{AUC}}$ in our LMM did not alter the significant interaction effect for FMD 1 hour following 3 different intensities of HG exercise $(P<0.01)$. 
223

224

225

226

227

229

230

231

232

233

234

235

236

237

238

239

240

241

242

\section{Discussion}

The purpose of the present study was to determine the effect of different intensities of handgrip exercise on post-exercise brachial artery endothelium-dependent conduit artery function. Previous research has produced conflicting findings pertaining to conduit artery functional responses to acute exercise, with increases (Johnson et al. 2012; Tinken et al. 2009; Tyldum et al. 2009; Zhu et al. 2010), decreases (Dawson et al. 2008; Goel et al. 2007; Jones et al. 2010; Llewellyn et al. 2012; Rognmo et al. 2008) and no change (Dawson et al. 2008; Harvey et al. 2005; Jones et al. 2010) reported. As recently reviewed, these disparate findings may be related to differences in experimental methodology (Dawson et al. 2013), idiosyncratic effects of distinct exercise modes and intensities (Birk et al. 2013; Dawson et al. 2013; Padilla et al. 2007) and/or the timing of post-exercise measurements. Another issue is that previous experiments have involved large muscle group "systemic" exercise bouts, which may induce complex localized, reflex and humoral responses. We adopted handgrip exercise in the present study in an attempt to understand the localized impacts of distinct exercise intensities on post-exercise FMD measures of artery function.

Our principal finding was that intensity of handgrip exercise modified the post-exercise FMD response, with the highest exercise intensity (15\% MVC) demonstrating a large and significant elevation in FMD, one hour post-exercise. The intensity-dependent effect on postexercise FMD we observed may be related to the impact of exercise-induced shear stress. Shear stress patterns are known to directly induce changes in the FMD/endothelial response to acute exercise (Green et al. 2005; Green et al. 2002; Laughlin et al. 2008; Thijssen et al. 2009c; Tinken et al. 2009). Elevated antegrade shear, in the absence of changes in retrograde shear, has been linked to improvement in endothelium-dependent vasodilation (Tinken et al. 2009), whilst increases in oscillatory shear and/or retrograde flow may be associated with 
increased endothelin-1 expression (Chappell et al. 1998; Himburg et al. 2007), the upregulation of reactive oxygen species (Hwang et al. 2003) and dose-dependent attenuation of endothelial function in humans (Schreuder et al. 2014; Thijssen et al. 2009c). In contrast to some forms of lower limb exercise, handgrip exercise is not associated with increases in retrograde flow and shear during exercise (see Figure 3), due largely to the decrease in distal vascular resistance (Green et al. 2005; Thijssen et al. 2009b). In contrast, there are large increases in retrograde shear during the early phases of leg cycling, which induces an oscillatory pattern of flow and shear in the brachial artery (Green et al. 2005; Green et al. 2002). When comparing shear rate patterns in the current study to our previous studies (Birk et al. 2013), clear differences can be observed in both the pattern and magnitude of shear responses to cycle versus handgrip exercise (Figure 3). It is plausible that the increase in antegrade shear observed, particularly at the highest handgrip exercise intensity in the present study, induces a post-exercise improvement in FMD. This would be consistent with what is known regarding the impacts of acute exercise stress on eNOS bioavailability and consequent chronic adaptation and upregulation (Hambrecht et al. 2003). Our data are also suggestive of a possible threshold effect of antegrade shear on artery function. We observed significantly higher antegrade shear at $15 \%$ compared to the other exercise intensities and the $15 \%$ condition was the only one associated with an elevation in FMD post exercise. Further studies will be required to address the idea of an 'antegrade shear threshold' and to examine the impact of shear rate manipulation during handgrip exercise on arterial adaptation in function and structure.

In the present study, we observed no reduction in FMD in the immediate post-exercise period in response to any intensity of HE. This contrasts with some previous studies examining the acute impacts of larger muscle group exercise on arterial function. Studies that have focused 
predominantly on leg exercise with multiple post-exercise FMD measures (Birk et al. 2013; Johnson et al. 2012), have typically observed a decrement in function immediately following exercise which appears to be larger at higher intensities. Both localized handgrip exercise and large muscle group exercise lead to a (dose-dependent) increase in brachial artery blood flow. Therefore, the distinct immediate post-exercise changes in FMD seen in studies of large muscle group exercise may relate to the impact of such exercise on retrograde shear, the production of reactive oxygen species (ROS) (Goto et al. 2003) or increases in adrenergic tone. However, as we did not assess oxidative stress or adrenergic tone in the present study, we cannot rule out impacts of handgrip exercise on these parameters. Our findings are therefore limited to the observation that handgrip and cycling exercise produce distinct immediate effects on artery function in humans compared to larger muscle group exercise and it seems reasonable to assume that larger muscle group exercise would have greater impact on humoral or neural responses.

In another previous study performed by our group, we observed increases in FMD after 30 mins of handgrip exercise, in keeping with the current results. However, in that experiment, the increase in FMD was observed immediately, rather than one hour after the cessation of the exercise bout. The magnitude of increase in SR during handgrip exercise in the Tinken $e t$ al. study was similar to that observed in response to the lowest exercise intensity (5\% MVC) in the current experiment, but FMD\% increased significantly in Tinken et al., whereas changes in the current study, though directionally consistent, were more modest. One possible explanation for this disparity relates to a minor difference in the exercise modality: although both experiments involved the same contractile frequency (30 per min), the study of Tinken et al. utilised isometric hand-gripping against a fixed handpiece and resistance, whereas in the current study subjects performed isotonic exercise using a custom built device 
that allowed movement in the handpiece during contraction. These findings therefore raise the intriguing possibility that differences may exist between modalities of exercise, even when identical muscle groups are involved. This finding adds to previous work which suggested that small differences in the rate of handgrip contraction can alter the FMD response (Gonzales et al. 2011). Future studies will be necessary to specifically address this question.

We utilized handgrip exercise as an experimental tool to isolate the localized effects of exercise and it was not our intention to study whether exercise training of the forearm might translate into systemic health benefits. Nonetheless, our results warrant future studies that are focused on the potential differences in factors that may contribute to the distinct changes in FMD following different modalities of training. Another limitation of our study is that we were unable to assess responses to an exogenous NO donor. Since it is possible that our strenuous exercise in the current experiment may have induced an up-regulation of endothelium-derived vasodilators or augmented smooth muscle cell sensitivity to NO, future studies should address the impact of exercise intensity on responses to glyceryl trinitrate.

In summary, our findings contribute to the existing literature in that we have studied, for the first time, the impact of distinct intensities of localized handgrip exercise on artery function. Impacts of such exercise on arterial function are less likely to have resulted from systemic, reflex or circulating factors than responses associated with lower limb large muscle group exercise (Birk et al. 2013). Further studies for the acute and chronic effects of different forms of exercise will be important to shed light on phenomena which relate initial physiological challenge to chronic adaptation in humans. 


\section{Acknowledgements}

324 We would like to thank the participants for their time in completing this research study.

\section{Funding}

327 Professor Green's research is supported by the National Health and Medical Research

328 Council (NHMRC): grant ID 1045204

329

\section{Ethical Approval}

331 All procedures performed in studies involving human participants were in accordance with 332 the ethical standards of the institutional and/or national research committee and with the 1964 333 Helsinki declaration and its later amendments or comparable ethical standards. Informed 334 consent was obtained from all individual participants included in the study.

\section{Disclosures}

337 The authors declare that they have no conflict of interest. 


\section{References}

Atkinson G, Batterham AM, Thijssen DH, Green DJ (2013) A new approach to improve the specificity of flow-mediated dilation for indicating endothelial function in cardiovascular research J Hypertens 31:287-291

Birk GK, Dawson EA, Batterham AM, Atkinson G, Cable T, Thijssen DH, Green DJ (2013) Effects of exercise intensity on flow mediated dilation in healthy humans Int J Sports Med 34:409-414 artery disease in a marathon runner Am J Cardiol 99:743-744

Gonzales JU, Thompson BC, Thistlethwaite JR, Scheuermann BW (2011) Association between exercise hemodynamics and changes in local vascular function following 
acute exercise Applied physiology, nutrition, and metabolism $=$ Physiologie appliquee, nutrition et metabolisme 36:137-144 doi:10.1139/H10-097

Goto C et al. (2003) Effect of different intensities of exercise on endothelium-dependent vasodilation in humans. Role of endothelium-dependent nitric oxide and oxidative stress Circulation 108:530-535

Green DJ (2009) Exercise training as vascular medicine: direct impacts on the vasculature in humans Exerc Sport Sci Rev 37:196-202

Green DJ, Bilsborough W, Naylor LH, Reed C, Wright J, O'Driscoll G, Walsh JH (2005) Comparison of forearm blood flow responses to incremental handgrip and cycle ergometer exercise: relative contribution of nitric oxide J Physiol 562:617-628

Green DJ, Cheetham C, Reed C, Dembo L, O'Driscoll G (2002) Assessment of brachial artery blood flow across the cardiac cycle: retrograde flows during lower limb exercise J Appl Physiol 93:361-368

Green DJ, Dawson EA, Groenewoud HM, Jones H, Thijssen DH (2014) Is flow-mediated dilation nitric oxide mediated?: A meta-analysis Hypertension 63:376-382

Green DJ, O'Driscoll G, Joyner MJ, Cable NT (2008) Exercise and cardiovascular risk reduction: time to update the rationale for exercise? J Appl Physiol 105:766-768

Hambrecht R et al. (2003) Regular physical activity improves endothelial function in patients with coronary artery disease by increasing phosphorylation of endothelial nitric oxide synthase Circulation 107:3152-3158

Harvey PJ, Picton PE, Su WS, Morris BL, Notarius CF, Floras JS (2005) Exercise as an alternative to oral estrogen for amelioration of endothelial dysfunction in postmenopausal women Am Heart J 149:291-297

Himburg HA, Dowd SE, Friedman MH (2007) Frequency-dependent response of the vascular endothelium to pulsatile shear stress Am J Physiol Heart Circ Physiol 293:645-653 
389

390

391

392

393

394

395

396

397

398

399

400

401

402

403

404

405

406

407

408

409

410

411

412

Hwang J et al. (2003) Pulsatile versus oscillatory shear stress regulates NADPH oxidase subunit expression: implication for native LDL oxidation. Circ Res 93:1225-1232

Johnson BD, Padilla J, Wallace JP (2012) The exercise dose affects oxidative stress and brachial artery flow-mediated dilation in trained men Eur J Appl Physiol 112:33-42

Jones H, Green DJ, George K, Atkinson G (2010) Intermittent exercise abolishes the diurnal variation in endothelial-dependent flow-mediated dilation in humans Am J Physiol Regul Intergr Comp Physiol 298:427-432

Joyner MJ, Green DJ (2009) Exercise protects the cardiovascular system: effects beyond traditional risk factors J Physiol 587:5551-5558

Laughlin MH, McAllister RM (1992) Exercise training-induced coronary vascular adaptation J Appl Physiol 73:2209-2225

Laughlin MH, Newcomer SC, Bender SB (2008) Importance of hemodynamic forces as signals for exercise-induced changes in endothelial cell phenotype J Appl Physiol $104: 588-600$

Llewellyn TL, Chaffin ME, Berg KE, Meendering JR (2012) The relationship between shear rate and flow-mediated dilation is altered by acute exercise Acta Physiol 205:394-402

Padilla J, Harris R, Fly A, Rink L, Wallace J (2006) The effect of acute exercise on endothelial function following a high-fat meal Eur J Appl Physiol 98:256-262

Padilla J, Harris RA, Wallace JP (2007) Can the measurement of brachial artery flowmediated dilation be applied to the acute exercise model? Cardiovascular Ultrasound $5: 45$

Padilla J, Simmons GH, Vianna LC, Davis MJ, Laughlin MH, Fadel PJ (2011) Brachial artery vasodilatation during prolonged lower limb exercise: role of shear rate Exp Physiol 96:1019-1027 
413

414

415

416

417

418

419

420

Rognmo O et al. (2008) Endothelial function in highly endurance-trained men: effects of acute exercise J Strength Cond Res 22:535-542

Schreuder TH, Green DJ, Hopman MT, Thijssen DH (2014) Acute impact of retrograde shear rate on brachial and superficial femoral artery flow-mediated dilation in humans Physiological reports 2:e00193

Takase B et al. (1998) Endothelium-dependent flow-mediated vasodilation in coronary and brachial arteries in suspected coronary artery disease. Am J Cardiol 82:1535-1539

Thijssen DHJ et al. (2011) Assessment of flow mediated dilation (FMD) in humans: a methodological and physiological guideline Am J Physiol Heart Circ Physiol 300:212

Thijssen DHJ et al. (2009a) Does arterial shear explain the magnitude of flow-mediated dilation?: a comparison between young and older humans. Am J Physiol Heart Circ Physiol 296:57-64

Thijssen DHJ, Dawson EA, Black MA, Hopman MTE, Cable NT, Green DJ (2009b) Brachial artery blood flow responses to different modalities of lower limb exercise. Med Sci Sports Exerc 41:1072-1079

Thijssen DHJ, Dawson EA, Tinken TM, Cable NT, Green DJ (2009c) Retrograde flow and shear rate acutely impair endothelial function in humans Hypertension 53:986 - 992

Thijssen DHJ et al. (2008) The impact of baseline diameter on flow mediated dilation (FMD) differs in young and older humans Am J Physiol Heart Circ Physiol 295:1594 - 1598

Tinken TM et al. (2009) Impact of shear rate modulation on vascular function in humans Hypertension 54:278-285

Tyldum GA, Schjerve IE, Tjonna AE, Kirkeby-Garstad I, Stolen TO, Richardson RS, Wisloff U (2009) Endothelial dysfunction induced by post-prandial lipemia: complete 
437

438

439

440

441

442

443

444

445

446

447

448

protection afforded by high-intensity aerobic interval exercise J Am Coll Cardiol 53:200-206

Williams MRI, Westerman RA, Kingwell BA, Paige J, Blombery PA, Sudhir K, Komesaroff PA (2001) Variations in endothelial function and arterial compliance during the menstrual cycle J Clin Endocrinol Metab 86:5389-5395

Woodman RJ et al. (2001) Improved analysis of brachial artery ultrasound using a novel edge-detection software system J Appl Physiol 91:929-937

Zhu W, Zeng J, Yin J, Zhang F, Wu H, Yan S, Wang S (2010) Both flow-mediated vasodilation procedures and acute exercise improve endothelial function in obese young men Eur J Appl Physiol 108:727-732 
Table 1. Comparisons between baseline FMD tests and pre-exercise resting shear rate and blood flow on each of the three testing days, with significance as $P \leq 0.05$.

\begin{tabular}{lcccc}
\hline \hline & $\begin{array}{c}\text { 5\% MVC } \\
\text { Handgrip }\end{array}$ & $\begin{array}{c}\text { 10\% MVC } \\
\text { Handgrip }\end{array}$ & $\begin{array}{c}\text { 15\% MVC } \\
\text { Handgrip }\end{array}$ & P Value \\
\hline Baseline FMD Test & & & & \\
Diameter (mm) & $3.82 \pm 0.42$ & $3.91 \pm 0.39$ & $3.89 \pm 0.58$ & $\mathbf{0 . 3 9}$ \\
FMD (\%) & $6.54 \pm 2.98$ & $6.41 \pm 1.64$ & $5.93 \pm 2.77$ & $\mathbf{0 . 7 3}$ \\
FMD (mm) & $0.02 \pm 0.01$ & $0.02 \pm 0.01$ & $0.02 \pm 0.01$ & $\mathbf{0 . 6 9}$ \\
Baseline mean $\mathrm{SR}_{\mathrm{AUC}}\left(10^{3} \cdot \mathrm{s}^{-1}\right)$ & $17.9 \pm 9.0$ & $16.6 \pm 7.7$ & $16.7 \pm 8.2$ & $\mathbf{0 . 8 2}$ \\
\hline \hline
\end{tabular}


Table 2. Hemodynamic parameters (5 min averages) at baseline and 30 minutes of unilateral handgrip exercise at distinct intensities (5, 10 and 15\% MVC) with corresponding one-way ANOVA effects for time and two-way ANOVA (Time 2 levels, Intensity 3 levels).

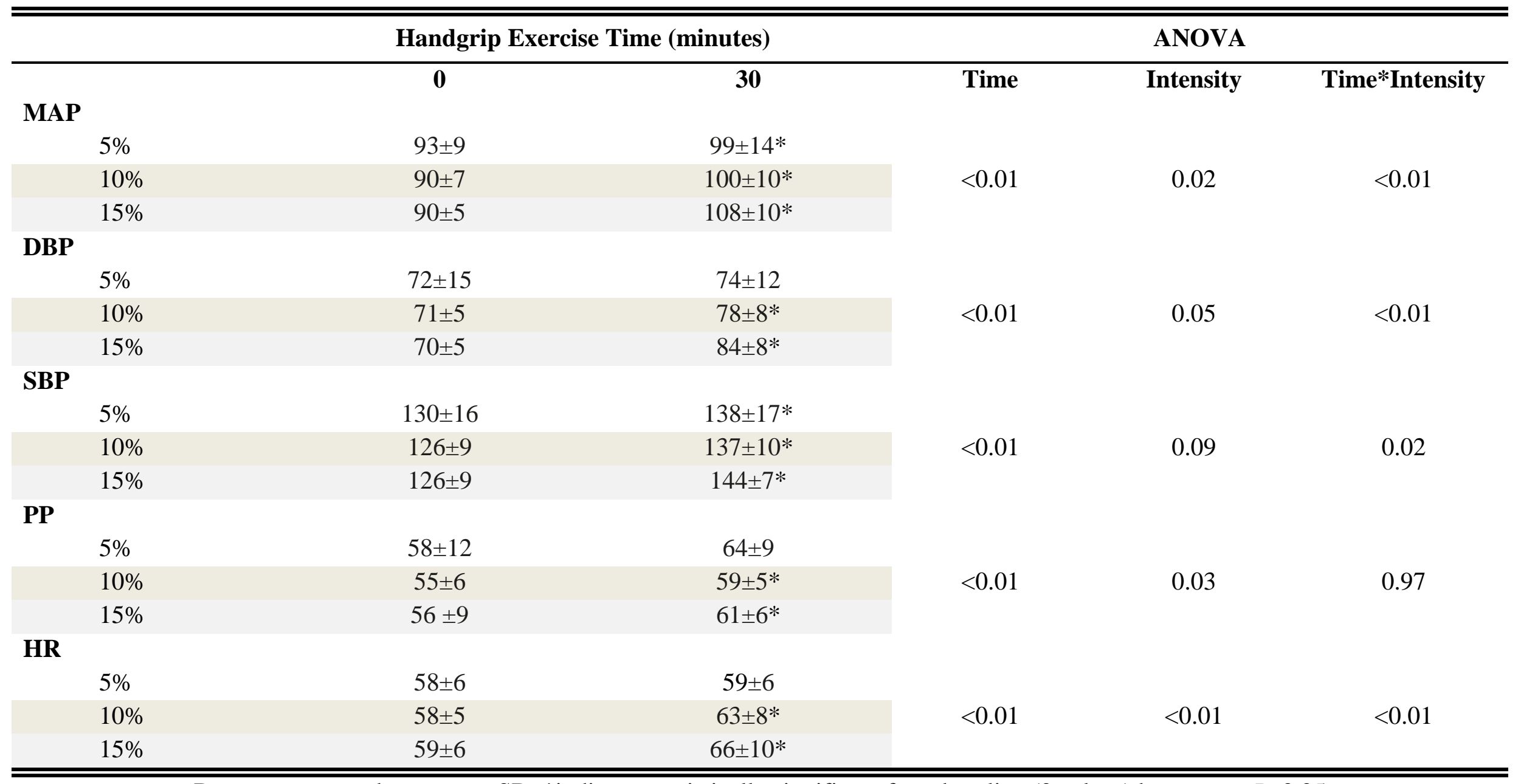


Table 3. Diameter, $\mathrm{SR}_{\mathrm{AUC}}$ and FMD responses pre, post and 60-minutes after cessation of 30 minutes of unilateral handgrip exercise at distinct intensities (5, 10 and $15 \%$ MVC).

\begin{tabular}{|c|c|c|c|c|c|c|c|c|c|c|c|c|}
\hline & \multicolumn{3}{|c|}{ 5\% MVC Handgrip } & \multicolumn{3}{|c|}{ 10\% MVC Handgrip } & \multicolumn{3}{|c|}{ 15\% MVC Handgrip } & \multicolumn{3}{|c|}{ 2-way ANOVA } \\
\hline & Pre & Post & $\begin{array}{c}\text { Post } \\
\text { 60mins }\end{array}$ & Pre & Post & $\begin{array}{c}\text { Post } \\
\text { 60mins }\end{array}$ & Pre & Post & $\begin{array}{c}\text { Post } \\
\text { 60mins }\end{array}$ & Intenstiy & Time & $\begin{array}{c}\text { Intensity } \\
* \text { Time }\end{array}$ \\
\hline $\begin{array}{l}\text { Diameter } \\
(\mathrm{mm})\end{array}$ & $3.8 \pm 0.4$ & $3.8 \pm 0.3$ & $3.9 \pm 0.5$ & $3.9 \pm 0.4$ & $4.0 \pm 0.5$ & $3.8 \pm 0.4 *$ & $3.9 \pm 0.6$ & $4.1 \pm 0.5^{*}$ & $3.8 \pm 0.4$ & 0.50 & $<0.01$ & 0.01 \\
\hline FMD (mm) & $0.02 \pm 0.01$ & $0.03 \pm 0.01 *$ & $0.02 \pm 0.01$ & $0.02 \pm 0.01$ & $0.03 \pm 0.01$ & $0.03 \pm 0.01$ & $0.02 \pm 0.01$ & $0.02 \pm 0.02$ & $0.04 \pm 0.02 *$ & 0.64 & 0.16 & 0.01 \\
\hline $\begin{array}{l}\left.\text { FMD SR } \text { AUC }_{\text {AU }} \cdot \mathrm{s}^{-1}\right) \\
\left(10^{3}\right.\end{array}$ & $17.9 \pm 9.0$ & $24.2 \pm 9.7 *$ & $17.2 \pm 7.7$ & $16.6 \pm 7.7$ & $30.0 \pm 14.1^{*}$ & $20.0 \pm 11.5$ & $16.7 \pm 8.2$ & $32.4 \pm 18.5^{*}$ & $17.3 \pm 9.0$ & 0.25 & $<0.01$ & 0.11 \\
\hline FMD (\%) & $6.5 \pm 3.0$ & $7.9 \pm 3.2$ & $5.9 \pm 3.1$ & $6.4 \pm 1.6$ & $6.6 \pm 3.1$ & $7.0 \pm 4.4$ & $5.9 \pm 2.8$ & $6.2 \pm 5.5$ & $10.4 \pm 5.8^{*}$ & 0.73 & 0.20 & 0.01 \\
\hline $\begin{array}{l}\text { FMD (\%) } \\
\text { ADJUSTED }\end{array}$ & $2.8 \pm 1.3$ & $3.4 \pm 1.3$ & $2.5 \pm 1.3$ & $2.7 \pm 0.7$ & $2.8 \pm 1.3$ & $3.0 \pm 1.8$ & $2.5 \pm 1.2$ & $2.6 \pm 2.3$ & $4.4 \pm 2.4^{*}$ & 0.48 & 0.04 & $<0.01$ \\
\hline
\end{tabular}

Data are presented as mean \pm SD. *indicates statistically significant from baseline values at $P \leq 0.05$ by $t$-test. FMD (\%) ADJUSTED values indicated FMD when statistically accounting for changes in baseline diameter and SR AUC using a Linear Mixed Model (LMM). 


\section{Figure Legends}

Fig. 1 a. Brachial artery antegrade shear rate before and during 30 minutes of handgrip exercise at 5\% (black bars), $10 \%$ (light grey bars) and 15\% (dark grey bars) MVC. b. Brachial artery retrograde shear rate before and during 30 minutes of handgrip exercise at $5 \%$ (black bars), 10\% (light grey bars) and 15\% (dark grey bars) MVC. Error bars represent SD.

Fig. 2 a. Change in FMD expressed as the immediate post-exercise FMD\% minus baseline FMD $\%$ at 5\% (black bars), 10\% (light grey bars) and 15\% (dark grey bars) MVC. b. Change in FMD expressed as the 60 minutes post-exercise FMD $\%$ minus baseline FMD $\%$ at 5\% (black bars), 10\% (light grey bars) and 15\% (dark grey bars) MVC. Error bars represent SD. Significance value $P<0.05$. * indicates statistical significance between highest (15\% MVC) and lowest (5\% MVC) intensity. ${ }^{\dagger}$ indicates statistical significance between moderate $(10 \%$ MVC) and highest (15\% MVC) intensity

Fig. 3 Brachial artery mean (average of the last 15mins) antegrade shear rate (black bars) during a. 30 minutes of handgrip exercise at 5, 10 and 15\% MVC, compared to b. 30 minutes of cycle exercise at 50, 70 and $85 \% \mathrm{VO}_{2}$ max. Brachial artery mean (average of the last 15mins) retrograde shear (grey bars) during c. 30 minutes of handgrip exercise at 5, 10 and $15 \%$ MVC, compared to d. 30 minutes of cycle exercise at 50, 70 and $85 \% \mathrm{VO}_{2}$ max. Error bars represent SD. 